\title{
Treatment of PC-3 cells with ultrasound combined with microbubbles induces distinct alterations in the expression of Bcl-2 and Bax
}

\author{
BAI WenKun, YANG ShaoLing, SHEN E, ZHANG JiZhen, SHEN ZhiYong \& HU Bing** \\ Shanghai Jiao Tong University Affiliated 6th People's Hospital, Shanghai Institute of Ultrasound in Medicine, Shanghai 200233, China
}

Received October 2, 2012; accepted November 17, 2012; published online May 22, 2013

\begin{abstract}
The objective of the present study was to investigate whether ultrasound combined with microbubbles induces apoptotic cell death in androgen-independent prostate cancer cells and to identify the probable mechanism. We used ultrasound in continuous wave mode with a frequency of $21 \mathrm{kHz}$ and a spatial-average temporal-average intensity of $46 \mathrm{~mW} / \mathrm{cm}^{2}$. Ultrasound combined with microbubbles ( $200 \mu \mathrm{L}$ SonoVue ${ }^{\mathrm{TM}}$ ) was used to treat androgen-independent human prostate cancer PC-3 cells for $30 \mathrm{~s}$. PC-3 cells were divided into three groups: the control group, the ultrasound group and the ultrasound combined with microbubbles group. Immediately after treatment, trypan blue exclusion was used to assess cell viability. Cell apoptosis at $24 \mathrm{~h}$ after treatment was measured using transmission electron microscopy and flow cytometry. Western blotting was used to evaluate the expression of the apoptosis-related proteins, Bcl-2 and Bax. Ultrasound combined with microbubbles had a minimal effect on the viability of PC-3 cells and induced minimal levels of cell lysis. The level of apoptosis in PC-3 cells induced by this modality was significantly higher than in controls $(12.77 \pm 0.31 \%$ vs. $2.56 \pm 0.22 \%, P<0.01)$. Treatment with ultrasound combined with microbubbles increased the expression of Bax, a pro-apoptotic protein, and decreased the expression of Bcl-2, an anti-apoptotic protein. It was concluded that ultrasound combined with microbubbles induces apoptotic cell death in human prostate cancer PC-3 cells through down-regulation of Bcl-2 and up-regulation of Bax.
\end{abstract}

low-frequency ultrasound, low-energy ultrasound, microbubble, apoptosis, Bcl-2, Bax

Citation: Bai W K, Yang S L, Shen E, et al. Treatment of PC-3 cells with ultrasound combined with microbubbles induces distinct alterations in the expression of Bcl-2 and Bax. Chin Sci Bull, 2013, 58: 3535-3540, doi: 10.1007/s11434-013-5753-y

In 2010 prostate cancer was ranked first in terms of the cancer incidence rate among American men, and its mortality rate was ranked second behind lung cancer [1]. The development of the androgen-independent stage of prostate cancer is believed to be primarily due to resistance to apoptosis, and it makes increase proliferation and survival of cells within primary or metastatic tumors [2]. Androgenindependent prostate cancer with anti-apoptotic properties, and it is difficult to be treated. The main therapeutic modalities used to treat prostate cancer are surgery, radiation and hormone therapy $[3,4]$. These modalities have been demonstrated to have certain curative effects as therapies for cancer, but they still have limitations such as injury to sur-

\footnotetext{
*Corresponding author (email: bing-hu021@hotmail.com)
}

rounding normal tissues, drug resistance and tumor recurrence [4-6].

Ultrasound is widely used for soft tissue imaging because of its perceived safety, noninvasiveness and low cost. It has also been used in therapy; in particular, it has been explored in some pre-clinical therapeutic studies and has been demonstrated to mediate apoptosis in many experimental systems in vitro or in vivo [7-9]. Cancer cells have been shown to be more susceptible than normal cells to ultrasound therapy $[10,11]$, a finding that was the experimental foundation for its application in the treatment of cancer. Moreover, the apoptosis effect induced in tumor by ultrasound can be enhanced by porphyrins, anticancer drugs and microbubbles $[12,13]$.

Clinically, ultrasound is described as being of low, me- 
dium or high intensity at values of $0-0.5,0.5-3$ and above 3 $\mathrm{W} / \mathrm{cm}^{2}$, respectively [14]. In the past, in studies involving ultrasound combined with microbubbles that demonstrated induced apoptosis in cancer cells, the authors usually used ultrasound frequencies between 1 and $4 \mathrm{MHz}$ [7,14]. As a result of the high energies involved in ultrasound treatment, cell lysis is the major phenomenon, and probably masks other effects on the surviving cells $[15,16]$. Frequencies below $1 \mathrm{MHz}$, and in particular at around $21 \mathrm{kHz}$, have seldom been studied; as a result, the mechanism involved in the induction of cell apoptosis remains unclear.

Bcl-2 belongs to a family of genes whose proteins play an important role in the regulation of apoptosis $[17,18]$. Bcl-2 is over-expressed in a variety of human cancers, including prostate cancer [19]. The Bcl-2 family can be classified into anti-apoptotic proteins and pro-apoptotic proteins. Bax, a pro-apoptotic protein in this family, in the apoptosis process it promote apoptosis of cell. Bcl-2, an anti-apoptotic protein, is known to have anti-apoptosis role in the apoptosis. In our study, we used an ultrasound frequency of 21 $\mathrm{kHz}$ with a spatial-average temporal-average intensity (ISATA) of $46 \mathrm{~mW} / \mathrm{cm}^{2}$. Low-frequency and low-energy ultrasound was combined with microbubbles to treat human prostate cancer PC-3 cells. After treatment, we evaluated the changes in Bcl-2 and Bax protein levels in apoptotic cells.

\section{Materials and methods}

This study was approved by the Ethics Committee of Shanghai Jiao Tong University Affiliated 6th People's Hospital, Shanghai Institute of Ultrasound in Medicine.

\subsection{Cell culture}

The androgen-independent human prostate cancer cell line,
PC-3, was obtained from the Cell Bank of the Chinese Academy of Sciences (Shanghai, China). The cells were grown in DMEM (GIBCO, Grand Island, NY, USA) supplemented with $10 \%$ heat-inactivated fetal bovine serum (Invitrogen, Carlsbad, CA, USA) at $37^{\circ} \mathrm{C}$ in humidified air containing $5 \% \mathrm{CO}_{2}$. In the study, PC-3 cells were resuspended at a density of $1 \times 10^{6}$ cells $/ \mathrm{mL}$ and were placed into $1.5 \mathrm{~mL}$ polystyrene test tubes. Each tube contained a $1 \mathrm{~mL}$ suspension of PC-3 cells. The diameter of the tubes was 13 $\mathrm{mm}$, and the tube bottoms were planar, which allowed them to be placed closer to the ultrasound probe.

\subsection{Ultrasound apparatus and microbubbles}

Ultrasound treatment was performed using a FS-450 ultrasonic processor (Shanghai Institute of Ultrasound in Medicine, 600 Yishan Road, Shanghai, China) in combination with SonoVue ${ }^{\mathrm{TM}}$ microbubble echo-contrast agent (Bracco SpA, Milan, Italy). The FS-450 ultrasonic processor was equipped with a built-in digital timer and an intensity regulator. The probe frequency was fixed at $21 \mathrm{kHz}$ and the intensity was $46 \mathrm{~mW} / \mathrm{cm}^{2}$. In all studies, ultrasound was generated by a $21 \mathrm{kHz}$ ultrasound probe using a continuous wave mode. The treatment time was $30 \mathrm{~s}$. The shape of the ultrasound probe was cylindrical and its diameter was 13 $\mathrm{mm}$, which was the same diameter as that of the test tubes. In all experiments, the clamp was attached to a metal stand to keep the transducer facing directly upward. The tube was placed in the center of the transducer with a gel interface (Figure 1). This setup allowed for standing wave formation due to the reflection of ultrasound radiation at the water-air interface [20].

The SonoVue ${ }^{\mathrm{TM}}$ agent used was a lipid-shelled ultrasound contrast agent composed of microbubbles that were filled with sulfur hexafluoride gas. The microbubbles were 2.5-6.0 $\mu \mathrm{m}$ in diameter. Before use, the SonoVue ${ }^{\mathrm{TM}}$ was reconstituted in $5 \mathrm{~mL}$ of phosphate-buffered saline (PBS) at

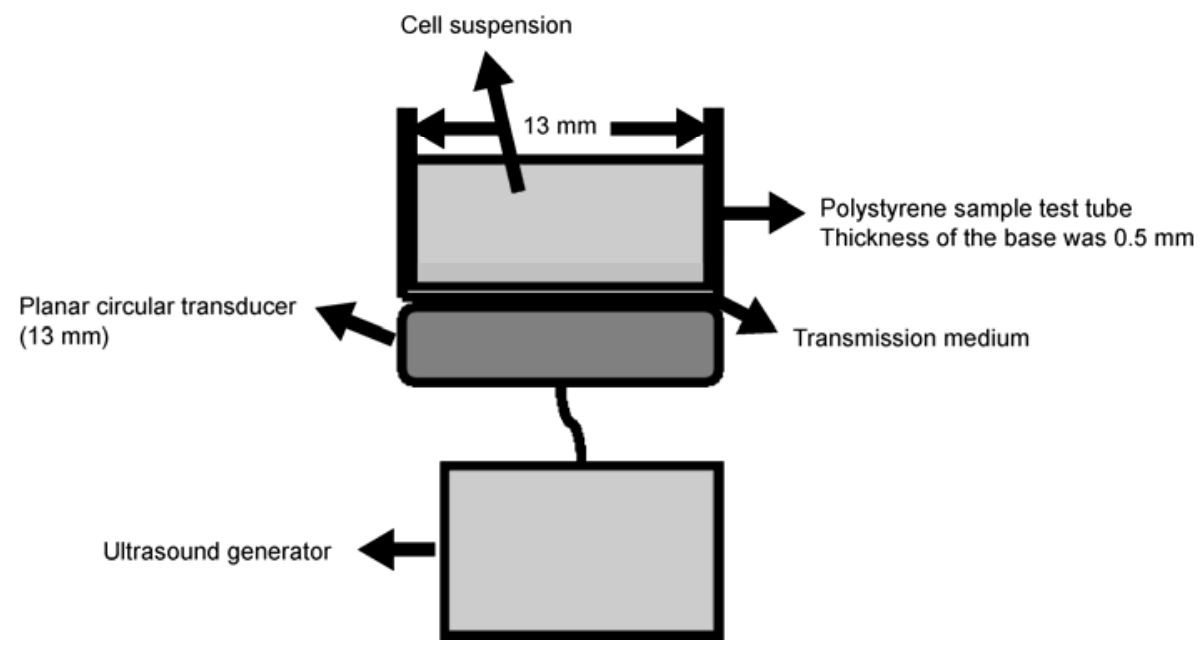

Figure 1 Experimental setup for ultrasound exposure. 
a concentration of $(2-5) \times 10^{8}$ microbubbles per $\mathrm{mL}$.

In all experiments, cells were divided into three groups: the control group (CT) that received no treatment; the ultrasound group (US), to which ultrasound treatment alone was administered; and the ultrasound combined with microbubbles group (US+MB), to which ultrasound combined with microbubbles ( $200 \mu \mathrm{L}$ SonoVue $\left.{ }^{\mathrm{TM}}\right)$ was administered. Each group contained six samples.

\subsection{Measurement of cell viability}

Cell viability was checked by trypan blue dye exclusion test. It was performed immediately after treatment by mixing $100 \mu \mathrm{L}$ of each cell suspension with an equal amount of $0.3 \%$ trypan blue solution (Sigma-Aldrich Chemical Co., St. Louis, MO, USA) in PBS [21]. After incubation for $5 \mathrm{~min}$, the numbers of stained and unstained cells were counted using a hemocytometer to estimate the number of intact non-viable and viable cells, respectively. The count before exposure was considered to be the $100 \%$ intact count, and the decrease in the number of intact cells after sonication was considered to be due to cell lysis [22].

\subsection{Measurement of cell proliferation}

After treatment, each group of cells was seeded at $3 \times 10^{3}$ cells/well in 96-well plates. After $24 \mathrm{~h}, 100 \mu \mathrm{L}$ of cell counting kit-8 (CCK8, Dojindo Laboratories, Kumamoto, Japan) was added. Plates were incubated for an additional $3 \mathrm{~h}$. The optical density for each well was measured using a microculture plate reader (Bio-Tek, Winooski, VT, USA) at a wavelength of $450 \mathrm{~nm}$ [23].

\subsection{Transmission electron microscopy}

After treatment, each group of cells was incubated for an additional $24 \mathrm{~h}$ in 6-well plates. Cells were observed for signs of apoptosis using transmission electron microscopy (TEM, Hitachi High-Technologies Corporation, Hitachis4800, Tokyo, Japan).

\subsection{Detection of apoptosis}

After ultrasound treatment, each group cells was incubated for an additional $24 \mathrm{~h}$ in 6-well plates. Cells were evaluated for apoptosis using FITC-labeled annexin $\mathrm{V}$ and propidium iodide (PI) double staining (Becton Dickinson, Franklin Lakes, NJ, USA) as previously described [24]. After $24 \mathrm{~h}$, treated and untreated cells were harvested, washed twice with PBS and resuspended with $0.5 \mathrm{~mL}$ PBS at a cell density of $1 \times 10^{6}$ cells $/ \mathrm{mL}$. Annexin V $(5 \mu \mathrm{L})$ and PI $(10 \mu \mathrm{L})$ were added to the wells in the dark. After incubation for 10 min, cells were analyzed by flow cytometry (Becton Dickinson) to determine the levels of apoptosis. The annexin V-FITC(+)/PI(-) cells were considered early apoptotic cells, while cells that were annexin V- FITC $(+) / \mathrm{PI}(+)$ were considered late apoptotic cells. Therefore, the total apoptotic cell count equaled the sum of the annexin-V-FITC(+)/PI(-) cells and the annexin-V-FITC(+)/ PI(+) cells [25].

\subsection{Western blot analysis}

Bcl-2 and Bax protein expression levels were measured using western blotting [26]. After $24 \mathrm{~h}$, treated and untreated cells were harvested and lysed, and supernatants were separated from the cell debris by centrifugation at 12000 rpm for $15 \mathrm{~min}$ at $4^{\circ} \mathrm{C}$. Aliquots containing $30 \mu \mathrm{g}$ of total protein were separated by SDS-PAGE and were transferred onto nitrocellulose membranes. The membranes were probed with primary rabbit monoclonal antibodies against Bcl-2 and Bax (Santa Cruz, San Diego, CA, USA) at $4^{\circ} \mathrm{C}$ overnight. Membranes were subsequently probed with a goat anti-rabbit secondary antibody conjugated with HRP (Santa Cruz) and visualized by ECL-based chemiluminescence. Protein band densities were quantified using Bio-Rad Quantity One software (Bio-Rad Laboratories, CA, USA).

\subsection{Statistical methods}

Data were expressed as the mean \pm SD. Different groups were compared using a paired $t$-test. A $P$ value of $<0.05$ was considered to be statistically significant.

\section{Results}

\subsection{Measurement of cell viability}

Loss of cell viability induced by treatment was investigated by performing trypan blue exclusion immediately after treatment (Figure 2). The total numbers of cells were almost constant in the US and US+MB groups. However, the fraction of intact non-viable cells tended to reach a plateau in the US+MB group.

\subsection{Measurement of cell proliferation}

Cell reproduction levels in the US and US+MB groups were significantly suppressed relative to the CT group $(P<0.01)$. This suppression was significantly greater in the US+MB group than in the US group $(P<0.01)$ (Figure 3$)$.

\subsection{Transmission electron microscopy}

The US and US+MB groups were found to have increased numbers of apoptotic cells; the number of apoptotic cells in the US+MB group was increased significantly. relative to the CT group $(P<0.01)$. The PC-3 cells exhibited the typical characteristics of apoptotic cells, such as nuclear condensation, cell shape, shrinkage and nuclear margination (Figure 4). 


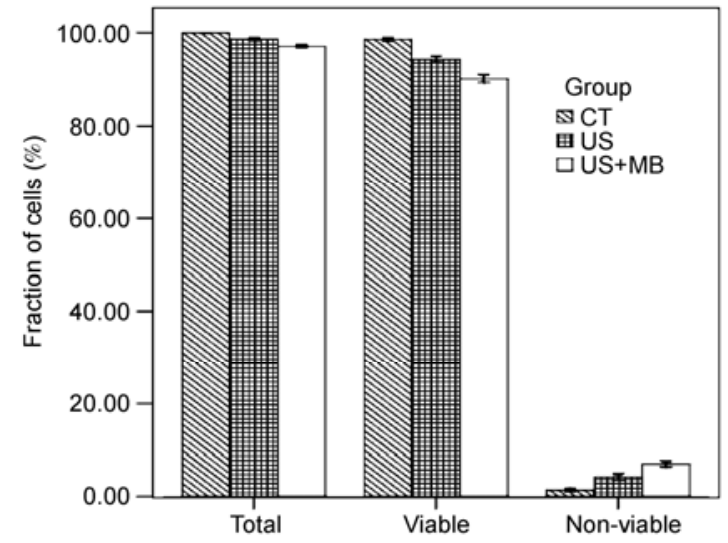

Figure 2 Effects of treatment with ultrasound and ultrasound in combination with microbubbles on cell viability. CT, Control group; US, ultrasound group; US+MB, ultrasound in combination with microbubbles group.

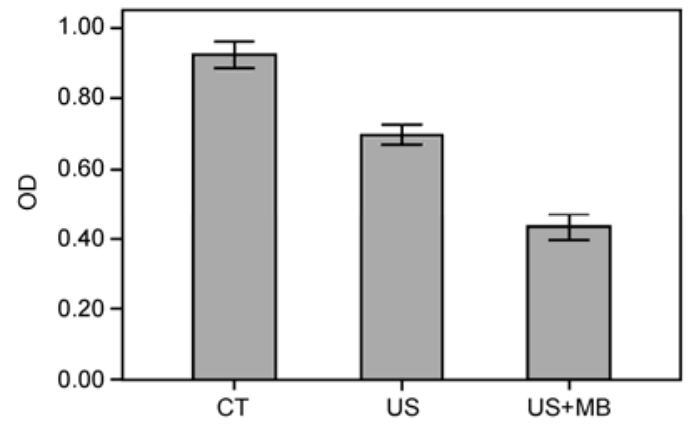

Figure 3 Cell proliferation in the CT, US and US+MB groups at $24 \mathrm{~h}$ after treatment. OD, Optical density; CT, control group; US, ultrasound group; US+MB, ultrasound in combination with microbubbles group.

\subsection{Detection of apoptosis}

As compared with the CT treatment group the US treatment group exhibited a slight apoptotic effect, and the US+MB treatment group exhibited an obvious apoptotic effect. These results demonstrated that apoptosis was indeed induced by both ultrasound and ultrasound combined with microbubbles. The use of microbubbles in combination with ultrasound further increased the apoptotic level, which was about five-fold higher than that in the CT group (Figure 5, Table 1).

\subsection{Western blot analysis}

Bcl-2 forms a heterodimeric complex with the apoptotic Bax protein, thereby neutralizing its apoptotic effects. Therefore, the ratio of $\mathrm{Bcl}-2 / \mathrm{Bax}$ is often considered a decisive factor in determining whether cells will undergo apoptosis or survive. We observed that cells treated with either ultrasound or ultrasound with microbubbles exhibited a

Table 1 Cell apoptosis in the CT, US and US+MB groups after ultrasound exposure

\begin{tabular}{cc}
\hline Group & Apoptosis (\%) \\
\hline CT & $2.56 \pm 0.22^{*}$ \\
US & $5.40 \pm 0.25^{\mathrm{a})}$ \\
US+MB & $12.77 \pm 0.31^{\mathrm{a}, \mathrm{b})}$ \\
\hline
\end{tabular}

* Mean \pm SD; a) $P<0.01$ vs. CT group; b) $P<0.01$ vs. US group. CT, Control group; US, ultrasound group; US+MB, ultrasound in combination with microbubbles group.
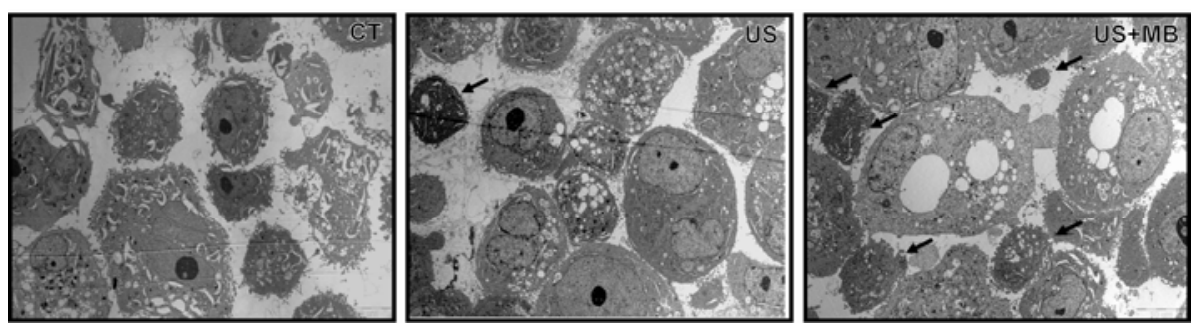

Figure 4 Apoptosis was increased in PC-3 cells at $24 \mathrm{~h}$ after treatment (magnification: $\times 1850$ ). CT, Control group; US, ultrasound group; US+MB, ultrasound in combination with microbubbles group.
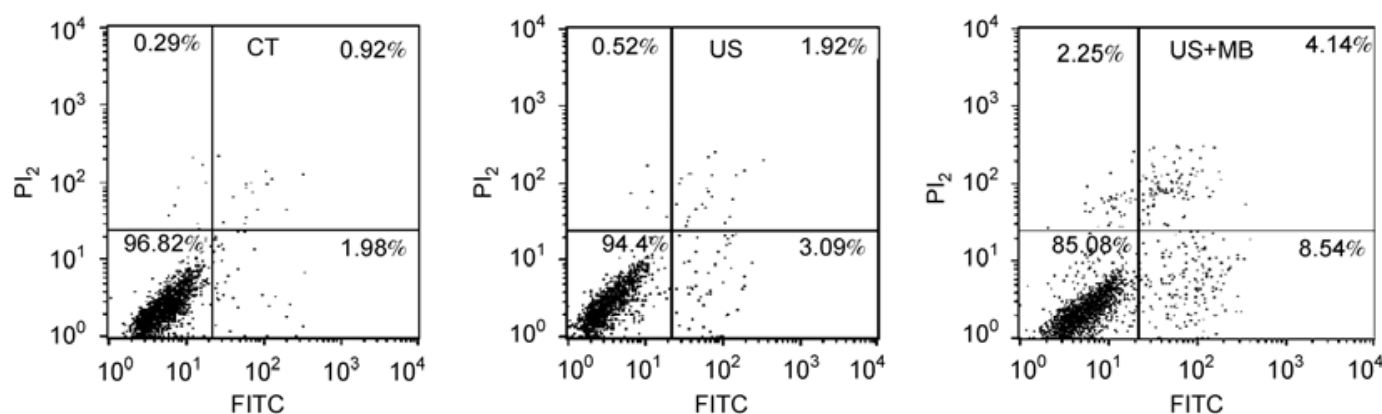

Figure 5 Apoptosis in PC-3 cells in the CT, US and US+MB groups at $24 \mathrm{~h}$ after treatment examined using flow cytometry. PI, Propidium iodide; FITC, flourescein iso-thiocyanate; CT, control group; US, ultrasound group; US+MB, ultrasound in combination with microbubbles group. 
decrease in Bcl-2 expression with a concomitant increase in Bax expression (Figure 6). This resulted in a substantial decrease in the $\mathrm{Bcl}-2 / \mathrm{Bax}$ ratio, which favored apoptosis (Figure 7). The US and US+MB groups exhibited a decrease in Bcl-2 expression with a concomitant increase in Bax expression, resulting in a substantial decrease in the Bcl-2/Bax ratio (US vs. CT, $P<0.01$; US+MB vs. CT, $P<$ $0.01)$. The US+MB group showed a more pronounced decrease in the Bcl-2/Bax ratio than did the US group (US+ MB vs. US, $P<0.01)$.

\section{Discussion}

The biophysical modes of ultrasound can be divided into three classes of effect: thermal, cavitational and mechanical [22]. The effects induced by low-frequency ultrasound are mainly mechanical and cavitational; the temperature increase through the thermal effect is negligible [27]. In the present study, we used regular cell media that was neither

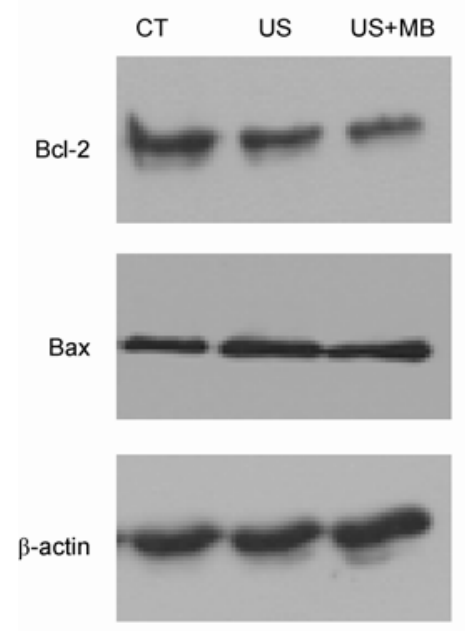

Figure 6 Expression of Bcl-2 and Bax in the CT, US and US+MB groups examined using western blotting. CT, Control group; US, ultrasound group; $\mathrm{US}+\mathrm{MB}$, ultrasound in combination with microbubbles group.

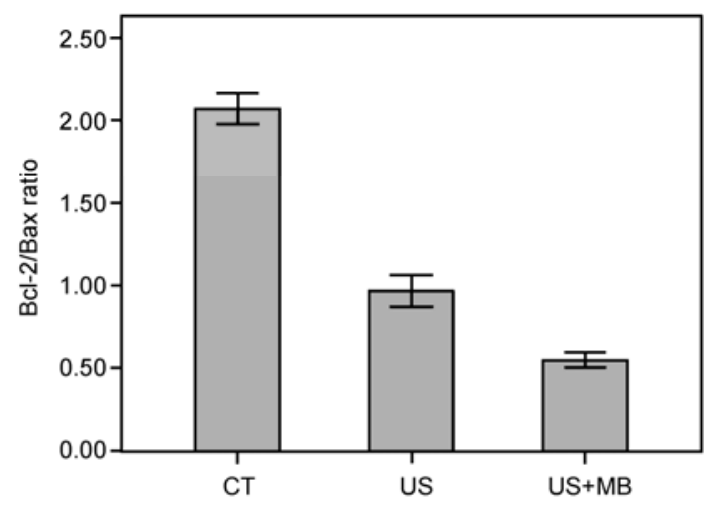

Figure 7 Expression of the Bcl-2/Bax ratio in the CT, US and US+MB groups. CT, control group; US, ultrasound group; US+MB, ultrasound in combination with microbubbles group. degassed nor air-saturated to avoid any resulting changes in the cells. Thus, the cell media may have contained air bubbles that could produce cavitation [28]. With exposure to ultrasound, microbubbles contained cavitation bubbles could produce intense collision, this energy could open the tight connection of the cell membrane. When microbubbles are added to the cell suspension, the cavitation effect is greater than when ultrasound is used alone. Collapsing microbubbles and the cavitation bubbles created by this collapse generate impulsive pressures, such as liquid jets and shock waves, which can cause cellular apoptosis. These pressures also affect neighboring cells the shock wave propagation distance is much larger than the radius of the cavitation bubbles [29].

In our study, we used low-frequency and low-energy ultrasound to treat androgen-independent prostate cancer PC-3 cells with ultrasound alone, and with ultrasound combined with microbubbles. Immediately after treatment, we evaluated cell viabilities. Our data indicated that ultrasound alone and ultrasound combined with microbubbles had minimal effects on PC-3 cell viabilities; these treatments also induced minimal cell lysis. We also measured cell proliferation at $24 \mathrm{~h}$ after ultrasound treatment. Our data indicated that ultrasound combined with microbubbles could suppress the proliferation of PC-3 cells. We also found that ultrasound combined with microbubbles could induce apoptosis in PC-3 cells. These data indicate that ultrasound combined with microbubbles inhibited cell proliferation using a mechanism involving the induction of apoptosis.

$\mathrm{Bcl}-2$, an anti-apoptotic protein of the Bcl-2 family, is known to contribute to neoplastic progression by inhibiting apoptosis to promote tumor development [30]. In most androgen-independent prostate cancers, Bcl-2 is overexpressed [2]. In preclinical prostate cancer models, inhibition of Bcl-2 expression probably increases apoptosis in the prostate cancer cells [31]. The Bax protein is pro-apoptotic; Bcl-2 has been shown to form a heterodimer complex with the pro-apoptotic member Bax, thereby neutralizing its pro-apoptotic effects. Therefore, the $\mathrm{Bcl}-2 / \mathrm{Bax}$ ratio is a decisive factor and plays an important role in determining whether cells will undergo death or will survive [32]. In the present study, ultrasound combined with microbubbles induced apoptosis in PC-3 cells, and western blot analysis showed that it affected the protein expression levels of Bcl-2 and Bax. These findings suggest that ultrasound combined with microbubbles up-regulates Bax and downregulates Bcl-2. Collectively, this may form the molecular basis for the apoptotic action in PC-3 cells.

In conclusion, ultrasound combined with microbubbles induced apoptotic cell death through the down-regulation of the anti-apoptotic Bcl-2 protein and the up-regulation of the pro-apoptotic Bax protein in prostate cancer PC-3 cells. Based on these results, ultrasound combined with microbubbles shows considerable promise as a treatment for androgen-independent prostate cancers. 
This work was supported by the National Natural Science Foundation of China (81271597) and the Major Infrastructure Projects of Shanghai Science and Technology (10JC1412600).

1 Jemal A, Siegel R, Xu J, et al. Cancer statistics, 2010. CA Cancer J Clin, 2010, 60: 277-300

2 Xu A H, Hu Z M, Qu J B, et al. Cyclic bisbibenzyls induce growth arrest and apoptosis of human prostate cancer PC3 cells. Acta Pharmacol Sin, 2010, 31: 609-615

3 Mangar S A, Huddart R A, Parker C C, et al. Technological advances in radiotherapy for the treatment of localised prostate cancer. Eur $\mathbf{J}$ Cancer, 2005, 41: 908-921

4 Tanaka G, Hirata Y, Goldenberg S L, et al. Mathematical modelling of prostate cancer growth and its application to hormone therapy. Philos Transact A Math Phys Eng Sci, 2010, 368: 5029-5044

5 Lecornet E, Ahmed H U, Moore C, et al. Focal therapy for prostate cancer: A potential strategy to address the problem of overtreatment. Arch Esp Urol, 2010, 63: 845-852

6 Baumert H. Salvage treatments for prostatic radiation failure. Cancer Radiothe, 2010, 14: 442-445

7 Tabuchi Y, Takasaki I, Zhao Q L, et al. Genetic networks responsive to low-intensity pulsed ultrasound in human lymphoma U937 cells. Cancer Lett, 2008, 270: 286-294

8 Tang W, Liu Q, Zhang J, et al. In vitro activation of mitochondriacaspase signaling pathway in sonodynamic therapy-induced apoptosis in sarcoma 180 cells. Ultrasonics, 2010, 50: 567-576

9 Feng Y, Tian Z, Wan M. Bioeffects of low-intensity ultrasound in vitro: Apoptosis, protein profile alteration, and potential molecular mechanism. J Ultrasound Med, 2010, 29: 963-974

10 Ashush H, Rozenszajn L A, Blass M, et al. Apoptosis induction of human myeloid leukemic cells by ultrasound exposure. Cancer Res, 2000, 60: 1014-1020

11 Rosenthal I, Sostaric J Z, Riesz P. Sonodynamic therapy-A review of the synergistic effects of drugs and ultrasound. Ultrason Sonochem, 2004, 11: 349-363

12 Tian Z, Quan X, Xu C, et al. Hematoporphyrin monomethyl ether enhances the killing action of ultrasound on osteosarcoma in vivo. $\mathrm{J}$ Ultrasound Med, 2009, 28: 1695-1702

13 Kolarova H, Tomankova K, Bajgar R, et al. Photodynamic and sonodynamic treatment by phthalocyanine on cancer cell lines. Ultrasound Med Biol, 2009, 35: 1397-1404

14 Jackson J K, Pirmoradi F N, Wan C P, et al. Increased accumulation of paclitaxel and doxorubicin in proliferating capillary cells and prostate cancer cells following ultrasound exposure. Ultrasonics, 2011, 51: 932-939

15 Kawai N, Iino M. Molecular damage to membrane proteins induced by ultrasound. Ultrasound Med Biol, 2003, 29: 609-614

16 Marentis T C, Kusler B, Yaralioglu G G, et al. Microfluidic sonicator for real-time disruption of eukaryotic cells and bacterial spores for
DNA analysis. Ultrasound Med Biol, 2005, 31: 1265-1277

17 Reed J C. Bcl-2 and the regulation of programmed cell death. J Cell Biol, 1994, 124: 1-6

18 Yang E, Korsmeyer S. Molecular thanatopsis: Adiscourse on the BCL2 family and cell death. Blood, 1996, 88: 386-401

19 Apakama I, Robinson M C, Walter N M, et al. Bcl-2 overexpression combined with $\mathrm{p} 53$ protein accumulation correlates with hormonerefractory prostate cancer. Br J Cancer, 1996, 74: 1258-1262

20 Hassan M A, Feril L B Jr, Suzuki K, et al. Evaluation and comparison of three novel microbubbles: Enhancement of ultrasound-induced cell death and free radicals production. Ultrason Sonochem, 2009, 16: 372-378

21 Lagneaux L, de Meulenaer E C, Delforge A, et al. Ultrasonic lowenergy treatment: A novel approach to induce apoptosis in human leukemic cells. Exp Hematol, 2002, 30: 1293-1301

22 Furusawa Y, Zhao Q L, Hassan M A, et al. Ultrasound-induced apoptosis in the presence of Sonazoid and associated alterations in gene expression levels: A possible therapeutic application. Cancer Lett, 2010, 288: 107-115

23 Sicklick J K, Li Y X, Jayaraman A, et al. Dysregulation of the Hedgehog pathway in human hepatocarcinogenesis. Carcinogenesis, 2006, 27: 748-757

24 Wang X B, Liu Q H, Wang P, et al. Enhancement of apoptosis by sonodynamic therapy with protoporphyrin IX in isolate sarcoma 180 cells. Cancer Biother Radiopharm, 2008, 23: 238-246

25 Fang H Y, Tsai K C, Cheng W H, et al. The effects of power on-off durations of pulsed ultrasound on the destruction of cancer cells. Int $\mathbf{J}$ Hyperthermia, 2007, 23: 371-380

26 Li Y C, Lin H J, Yang J H, et al. Baicalein-induced apoptosis via endoplasmic reticulum stress through elevations of reactive oxygen species and mitochondria dependent pathway in mouserat hybrid retina ganglion cells (N18). Neurochem Res, 2008, 34: 418-429

27 Samuel S, Miller D L, Fowlkes J B. The relationship of acoustic emission and pulse-repetition frequency in the detection of gas body stability and cell death. Ultrasound Med Biol, 2006, 32: 439-447

28 Chumakova O V, Liopo A V, Evers B M, et al. Effect of 5-fluorouracil, optison and ultrasound on MCF-7 cell viability. Ultrasound Med Biol, 2006, 32: 751-758

29 Kodama T, Tomita Y, Koshiyama K, et al. Transfection effect of microbubbles on cells in superposed ultrasound waves and behavior of cavitation bubble. Ultrasound Med Biol, 2006, 32: 905-914

30 Tsujimoto $\mathrm{Y}$, Croce C M. Analysis of the structure, transcripts, and protein products of bcl-2, the gene involved in human follicular lymphoma. Proc Natl Acad Sci USA, 1986, 83: 5214-5218

$31 \mathrm{Ku} \mathrm{J} \mathrm{H,} \mathrm{Seo} \mathrm{S} \mathrm{Y,} \mathrm{Kwak} \mathrm{C,} \mathrm{et} \mathrm{al.} \mathrm{The} \mathrm{role} \mathrm{of} \mathrm{survivin} \mathrm{and} \mathrm{Bcl-2} \mathrm{in}$ zinc-induced apoptosis in prostate cancer cells. Urol Oncol, 2013, in press

32 Malik A, Afaq S, Shahid M, et al. Influence of ellagic acid on prostate cancer cell proliferation: A caspase-dependent pathway. Asian Pac J Trop Med, 2011, 4: 550-555

Open Access This article is distributed under the terms of the Creative Commons Attribution License which permits any use, distribution, and reproduction in any medium, provided the original author(s) and source are credited. 